\title{
WhatsApp como Recurso Educativo y Tecnológico en la Educación
}

\section{WhatsApp as an Educational and Technological Resource in Education}

\author{
Charo Mimi Cervantes Rosas ${ }^{1}$ \\ https://orcid.org/0000-0002-8564-6067 \\ Universidad César Vallejo, Perú \\ Cleofé Genoveva Alvites-Huamaní ${ }^{2}$ \\ https://orcid.org/0000-0001-6328-6470
}

Recibido: 17-05-2021

Aceptado: 30-08-2021

\section{Cita Recomendada}

Cervantes, C. y Alvites-Huamaní, C. (2021). WhatsApp como recurso educativo y tecnológico en la educación. Hamut'ay, 8 (2), 69-78, http://dx.doi.org/10.21503/hamu.v8i2.2294

\section{Resumen}

En este estudio se hace una revisión documental del WhatsApp en el ámbito educativo en las bases de datos de SCOPUS, WOS, Scielo y Cybertesis, encontrándose un total de 101 documentos. Después de considerar los criterios de inclusión y exclusión, la muestra documental quedo conformada por 35 artículos, que fueron analizados desde una perspectiva teórico-conceptual para conocer cómo se originó y evolucionó el WhatsApp como es usado como recurso tecnológico; qué funcionalidades tiene como recurso educativo y cuáles son sus ventajas y desventajas en la educación.

Se encontró que aunque el WhatsApp no fue diseñado para el entorno educacional, se volvió uno de los medios más utilizados en el proceso de enseñanza-aprendizaje, por las posibilidades que abre a la construcción de conocimiento, y su facilidad de uso, acceso y vinculación a funcionalidades como el chat grupal e individual, la mensajería instantánea, los mensajes de voz, las llamadas y videollamadas, el compartir documentos, fotos, imágenes, videos, audios y una diversidad de páginas web, entre las más destacadas; funcionalidades con las que se puede promover la participación de estudiante-estudiante y docente-estudiante, la lectura de textos en diferentes formatos, el realizar evaluaciones diagnósticas, o el debatir sobre un tema en específico; bajo la orientación que el docente realice.

Sin embargo, a pesar de las potencialidades del WhatsApp, también se debe poner atención sobre los impactos negativos que puede generar en el desempeño de cada estudiante, sino se cuenta con la guía del docente y de los padres, ni se establecen normas para su uso como medio, debido a que esta tiene acceso abierto e ilimitado.

Palabras clave: WhatsApp, recurso tecnológico, red social, recurso educativo, TIC.

1. Estudiante del Doctorado en educación de la Universidad César Vallejo-SP, Magíster en educación, licenciada en educación. Estudiante adscrita al proyecto PIPD, Innovación docente y uso de las Tecnologías de la Información y Comunicación en el proceso de enseñanza-aprendizaje. E-mail: chamimicero@gmail.com

2. Investigador Renacyt-CONCYTEC. Docente investigador de Posgrado.cleoalvitesh@gmail.com 


\begin{abstract}
In this study, a documentary review of WhatsApp in the educational field it is made in the databases of SCOPUS, WOS, Scielo and Cybertesis, finding a total of 101 documents. After considering the inclusion and exclusion criteria, the documentary sample was made up of 35 articles, which they were analyzed from a theoretical-conceptual perspective to find out how WhatsApp originated and evolved; how it is used as a technological resource; what functionalities it has as an educational resource and what are its advantages and disadvantages in education.

It was found that although WhatsApp was not designed for the educational environment, it became one of the most used means in the teaching-learning process, due to the possibilities it opens up to construe knowledge, and its ease of use, access and linkage functionalities such as group and personal chat, instant messaging, voice messages, calls and video calls, sharing documents, photos, images, videos, audio and a variety of web pages, among the most prominent; functionalities with which it is possible to promote student-student and teacher-student participation, reading texts in different formats, conducting diagnostic evaluations, or discussing a specific topic; under the guidance of the teacher.

However, despite the potential of WhatsApp, attention must also be paid to the negative impacts that it can generate on the performance of each student, if it does not have the guidance of the teacher and parents, nor are rules established for its use. as a medium, because it has open and unlimited access.
\end{abstract}

Keywords: WhatsApp, technological resource, social network, educational resource, ICT.

\section{Introducción}

Los cambios forzados sufridos por el mundo en la actualidad, dada la coyuntura que está atravesando a raíz de la pandemia COVID-19, se evidencia en diferentes sectores de la humanidad, en la transformación de los estilos de vida y las rutinas que se tenían, lo que ha implicado el tener que aprender a vivir bajo el aislamiento obligatorio, la búsqueda de nuevas alternativas de interacción y el refuerzo de otras para continuar con las labores personales y profesionales, en la que las Tecnologías de la Información y la Comunicación (TIC) tienen una preponderancia. Dentro de este panorama la educación también ha tenido que asumir el reto de una enseñanza virtualizada, apropiándose de diversas herramientas y recursos tecnológicos que faciliten su desarrollo (Oliveira et al., 2020). En un contexto todavía incierto, donde el retorno a la presencialidad por parte de las instituciones educativas de todos los niveles no se avizora, sehan replanteado aspectos del trabajo convencional y de la educación presencial análoga a partir del fortalecimiento de las interacciones virtuales; desplegándose acciones para dar continuidad a la enseñanza y al aprendizaje, en el que las TIC asumen un rol muy significativo en el progreso de muchas actividades educativas y profesionales; de esta manera los docentes y otros profesionales, asumen la necesidad de utilizarlas para cumplir con sus respectivos trabajos (UNESCO, 2021).

Es así que, muchas instituciones y organizaciones buscaron alternativas fáciles y accesibles para sus usuarios, para que estos no perdieran el vínculo que tenían con la presencialidad análoga; es en este contexto donde emerge el WhatsApp para la educación, al ser de fácil acceso a gran multitud de usuarios y al abrir posibilidades de propagación ilimitada de información para diferentes áreas del desempeño humano. Por ello, en este estudio la revisión documental (Moreno et al., 2018), se ha propuesto como objetivos: i. Conocer cómo se originó y evolucionó el WhatsApp. ii. Indagar cómo el WhatsApp es usado como recurso tecnológico en el ámbito educativo. iii. Reconocer las funcionalidades del WhatsApp como recurso educativo. iv. Describir las ventajas y desventajas de WhatsApp como recurso educativo. 


\section{Método}

El estudio forma parte de una revisión documental en la que se realiza un análisis teóricoconceptual de artículos científicos explorados con rigurosidad en bases de datos reconocidas como SCOPUS, WOS, Scielo y Cybertesis. Los descriptores referidos fueron: WhatsApp como recurso educativo, uso del WhatsApp en el ámbito educativo, ventajas y desventajas del WhatsApp como recurso (Román, Marín y Peirats, 2021). Para la muestra de estudio documental se tuvo como criterios de inclusión y exclusión (Tabla 1).

Tabla 1. Criterios de inclusión y exclusión para la revisión de la literatura

\begin{tabular}{clrl}
\hline Criterios de inclusión & & Criterios de exclusión \\
\hline a. & $\begin{array}{l}\text { Artículos de revistas científicas, } \\
\text { actas de congreso, tesis. }\end{array}$ & f. & Libros, capítulos de libros. \\
\hline b. $\begin{array}{l}\text { Publicaciones de los 4 últimos } \\
\text { años (2018-2021) }\end{array}$ & g. & $\begin{array}{l}\text { Publicaciones mayores a los } \\
\text { últimos 5 años }\end{array}$ \\
\hline c. $\begin{array}{l}\text { Publicaciones en español, inglés } \\
\text { y portugués }\end{array}$ & h. & $\begin{array}{l}\text { Publicaciones en alemán, } \\
\text { francés, chino entre otros. }\end{array}$ \\
\hline d. $\begin{array}{l}\text { Publicaciones de acceso abierto } \\
\text { a texto completo. }\end{array}$ & i. & $\begin{array}{l}\text { Publicaciones de acceso } \\
\text { restringido }\end{array}$ \\
\hline e. & Artículos originales & j. & $\begin{array}{l}\text { Artículos de revisión, y/o } \\
\text { artículos duplicados. }\end{array}$ \\
\hline
\end{tabular}

Fuente: Elaboración propia (2021).

Con la finalidad de cumplir con los objetivos de la investigación, se siguió una secuencia de fases para recolectar la información bibliográfica y posterior análisis narrativo y descriptivo de la revisión de la literatura, lo cual se describe en la Figura 1.

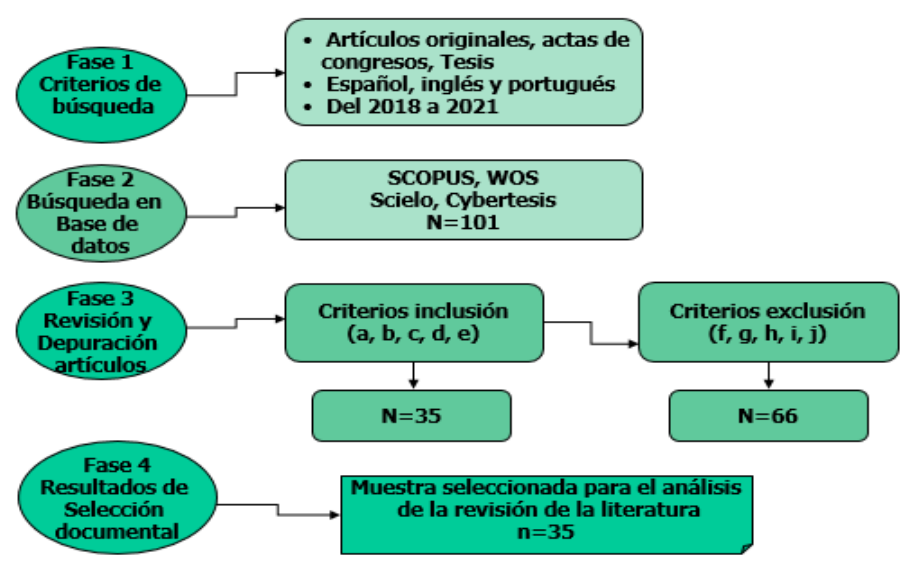

Figura 1. Secuencia de fases para recolectar la información bibliográfica Fuente: Elaboración propia (2021).

\section{Origen y evolución de WhatsApp}

WhatsApp inició su funcionamiento el año 2009, como una red social exclusiva para la marca IPhone, con la funcionalidad de ser un sistema de mensajería simple. Fue fundada por Jan Kuom nativo de Ucrania y Brian Acton de Estados Unidos que tenían antecedentes laborales en empresas de renombre como Adobe, Yahoo y Apple. Tapia-Repetto, Gutierrez y TremilloMaldonado, (2019) afirman que el "término WhatsApp proviene de un juego de palabras y adaptación de las palabras "What's up", una frase coloquial inglesa que significa ¿Qué pasa?, y App, la abreviatura también inglesa de aplicaciones" (p. 39). Su uso se ha dado primeramente como medio de socialización, con conversaciones vagas por parte de sus consumidores. Mejía (2019) indica que la tecnología ha demostrado ser una gran herramienta en todo el proceso de evolución de las sociedades modernas, al mejorar los procesos comunicativos y al revolucionar las estrategias de interconexión de las personas que lo utilizan de acuerdo a sus necesidades.

En el año 2014 la aplicación WhatsApp debido a su gran crecimiento y aceptación por parte de la comunidad juvenil, fue vendida a la empresa Facebook, una de las más grandes compañías en el rubro tecnológico. Esta red WhatsApp suma algo de 1.500 millones de personas aproximadamente en 180 países, quienes lo utilizan no solo en el celular, sino también a través de la web en una computadora de escritorio (Pérez et al., 2020). WhatsApp se encuentra en segundo lugar de uso de las redes sociales más requeridas en el mundo (Mas, 2019), y su uso se ha extendido globalmente como medio de comunicación para diferentes actividades, al facilitar diálogos en grupo o de manera individual con muchas personas al mismo tiempo, al compartir videos, fotos o las mismas transmisiones en vivo, funcionalidades que hacen que el usuario se sienta satisfecho con respecto a otras redes sociales (Suárez-Lantarón, 2018). 
WhatsApp como recurso tecnológico en el ámbito educativo

El quehacer educativo se desarrolla en torno a diferentes propuestas virtuales, que se incorporan como parte de la cotidianidad en las sesiones de aprendizaje, y como estrategia de los docentes para diferentes actividades académicas (Marín-Díaz y Cabero-Almenara, 2019). Esto ha obligado a que se busquen recursos o medios que permitan llegar a la diversidad de estudiantes, lo que requiere de maestros que sepan manejar estas tecnologías virtuales (Safiatur, 2020), que capten su curiosidad y promuevan experiencias significativas, que orienten el uso de aplicaciones que tienen gran demanda por los jóvenes (Ruvalcaba et al., 2019). De esta manera es posible cambiar patrones de comportamiento que sean beneficiosos para el logro de aprendizajes y el desarrollo de contenidos en determinadas asignaturas, en donde los estudiantes pueden informarse a través de Internet, además de ser capaces de analizar, reflexionar y realizar síntesis para un determinado tema de estudio (LafaurieMolina, Sinning-Ordóñez y Valencia-Cobo, 2018).

Por ende, las TIC disponibles en Internet han cobrado protagonismo en las diferentes áreas de desempeño de los seres humanos, una de ellas es la mensajería instantánea, dentro del cual está el Whatsapp como apoyo imprescindible en el desarrollo social de los estudiantes, como medio de información e intercambio de comunicación (Cascales et al., 2020), como instrumento de apoyo para un mejor rendimiento académico por sus funcionabilidades dinámicas e interactivas (Alfarah y Bosco, 2018).

El trabajo académico a través de WhatsApp reta a los docentes a estar actualizados digitalmente (Mayor-Buzón, García-Pérez y RebolloCatalán, 2019), a estar familiarizados con sus funcionalidades y su conectividad, a conectarse desde cualquier punto del planeta y compartir información de interés, a trabajar de manera colaborativa con los padres de familia en beneficio de los estudiantes (Corona, 2020). Como funcionalidades para estudiantes, maestros y padres de familia se tienen: los chats grupales, las aperturas de sala, la comunicación síncrona y asíncrona, los videos, las fotos y el intercambio constante de información; características que lo han llevado rápidamente a insertarse en el sector educativo, volviéndose una necesidad para la comunicación e intercambio de información dentro del ámbito escolar (Crisol-Moya, HerreraNieves y Montes-Soldado, 2020).

La exploración de las TIC de vanguardia resulta muy conveniente en el trabajo escolar, al propiciar nuevas estrategias $y$ formas innovadoras de generar aprendizajes (FitriRahmadi, 2020), al generar entornos que llaman la atención de los educandos y padres de familia, por mostrarse como un recurso interesante, divertido y ventajoso para el proceso formativo y brindar experiencias acordes a la época actual, con tecnologías modernas y al alcance de todos (Escobar-Mamani y Gómez-Arteta, 2020); lo que facilita la acción académica por la gran variedad de bondades y beneficios que poseen, sobre todo que puede ser utilizado desde los más pequeños hasta los mayores.

WhatsApp es una aplicación que permite a estudiantes y familias organizarse de manera independiente y autónoma, ya sea en la formación de equipos de trabajo, en el potenciar la interacción a través de su espacio virtual, en cumplir las metas educativas y al mismo tiempo, fortalecer las relaciones interpersonales (VeytiaBucheli y Bastidas-Terán, 2020). Asimismo, constantemente atrae a nuevos usuarios por ser un servicio ofrecido con gratuidad por muchos operadores de telefonía celular, situación que ayuda a incrementar la comunicación e interconexión de manera colaborativa y cooperativa por parte de los usuarios de mensajería (Shahid \& Shaikh, 2019).

El WhatsApp es un sistema de comunicación imprescindible en la rutina de los nativos digitales y los usuarios que tienen la necesidad de hacer uso de ella por sus características únicas (Molina-García, 2020), por su utilidad en momentos de aislamiento total de las personas, en que las labores presenciales análogas quedaron interrumpidas a raíz de la pandemia, como único 
recurso académico para muchos docentes que innovaron el aprendizaje mediante la aplicación basada en la mensajería instantánea que tiene el WhatsApp (Paredes, 2020).

\section{Funcionalidades de WhatsApp como recurso educativo}

Al virtualizarse la educación muchos docentes se vieron obligados a buscar recursos tecnológicos como el WhatsApp que fue un medio para orientar el proceso de enseñanza-aprendizaje, con bondades al alcance de toda la comunidad académica, la mensajería instantánea grupal e individual, las llamadas de voz, las video llamadas, el permitir cargar archivos en variados formatos, carga de videos, audios, imágenes y fotografías en línea, todo esto agregado a una apertura de emoticones e imágenes que suplantan la escritura de una reacción. Así, la inmediatez de interacción a través de mensajes gratuitos, permitió compartir archivos y una serie de recursos, mensajes de voz; además de brindar otras alternativas como el realizar videollamadas, nacionales o internacionales; el personalizar las configuraciones; el sincronizar con la computadora en WhatsApp web y escritorio; la seguridad predeterminada que protege conversaciones; el permitir hasta 100MB para compartir recursos con gran facilidad (WhatsApp, 2021).

Por su versatilidad se ha convertido en estrategia para dar continuidad educativa y extender las actividades académicas a espacios poco usados con fines pedagógicos (Escofet, 2020). Esto exige que el docente encargado de su uso, lo maneje en forma planificada y apropiada, reflexione sobre su práctica y analice si está cumpliendo con los requerimientos de sus estudiantes y las familias; es por ello que requiere autocapacitarse para estar acorde con las nuevas tendencias digitales y no perder su autoridad, y esta no perturbe el proceso de aprendizaje (Sola-Reche, GarcíaVidal y Ortega-Navas, 2019). Dentro de sus responsabilidades está el brindar pautas que permitan una convivencia virtual adecuada y que se le otorgue el uso para los fines creados, tarea en la que es importante involucrar a los padres de familia en su seguimiento y el fijar horarios para las actividades académicas propuestas de acuerdo a la edad de los estudiantes (Malo-Cerrato, Martin-Perpina y Vinas, 2018). Es así que es muy importante direccionar el uso de WhatsApp para lograr desarrollar aprendizajes en un futuro, como parte no solo de una enseñanza netamente virtual sino también como complemento de la educación presencial análoga.

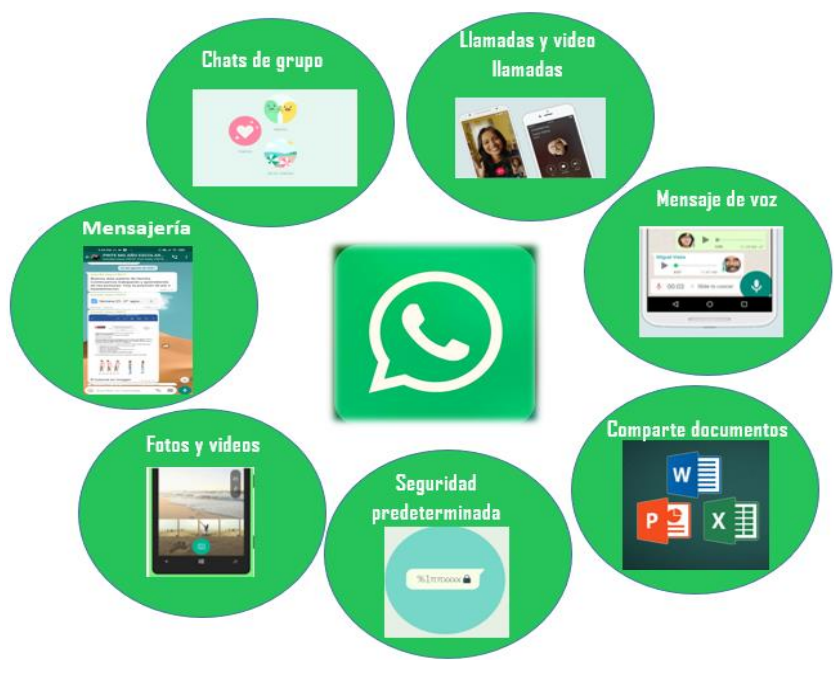

Figura 3. Funciones académicas de WhatsApp, basado Web Whatsapp.com (2021)

Fuente: elaboración propia (2021)

Ventajas educativas del WhatsApp

A pesar que WhatsApp nació con una finalidad muy ajena a la formación y su uso se difundió más como plataforma de distracción y esparcimiento social, la pandemia le dio un giro abismal a su uso, convirtiéndolo en un aliado para el ámbito educativo, en el que brinda variadas ventajas como facilidad de uso, acceso desde una diversidad de celulares, rapidez en la interconexión, disponibilidad en todo horario, funcionalidades variadas y oportunidad para colaborar, interactuar y compartir experiencias en diferentes ámbitos (Colmenares, 2021).

Cabe destacar que las ventajas de WhatsApp están condicionadas a un uso adecuado y a las mejoras constantes que brinda, circunstancias que permiten la producción de conocimiento y el desarrollo de un panorama educativo eficaz con temas de aprendizaje de interés común 
para la comunidad educativa (MaldonadoBerea, García-Gonzales y Sampedro-Requena, 2019); la formación de grupos de trabajo con afinidades, en los que se comparte información de interés, se fortalece la cooperación entre estudiantes, se mejora y crea más confianza con los docentes, estudiantes y padres de familia, se establece un abordaje más personalizado de la enseñanza, se motiva la adquisición de nuevos aprendizajes, se realiza un acompañamiento más individualizado, se promueve la participación de los integrantes más introvertidos y poco comunicativos, se incentiva a la imaginación durante las interacciones, se promueve la lectura de variados textos, se accede a materiales formativos en diferentes formatos, se comparten clases grabadas oralmente en formato de audio o video.

Además, WhatsApp permite la creación de un ambiente para exponer y expresarse sin ninguna restricción, que facilita a los docentes actividades como la evaluación diagnóstica sobre los aprendizajes, el desarrollo de una adecuada comunicación y expresión escrita, el fomento al trabajo colaborativo como apoyo académico entre estudiantes, la información inmediata sobre asuntos educativos, la organización y extensión de la labor del docente más allá del aula, la aclaración de dudas y la respuesta a interrogantes, la disponibilidad del docente en cualquier lugar y hora adecuada, la realimentación y finalmente la mejoría del rendimiento académico de los estudiantes (Suárez-Lantarón, 2018).

Desventajas de WhatsApp como recurso educativo

Así como ofrece ventajas, WhatsApp puede propiciar desventajas dada la masificación a la que está expuesta por la cantidad de personas en interacción grupal dentro de un aula, frente a lo que se requiere fijar normas desde un inicio que fomenten conductas y acciones adecuadas basadas en el respeto, para un buen desarrollo de las actividades académicas (Montilla, 2020).

Se ha observado que la generación de nativos digitales hacen uso constante del WhatsApp en edades muy tempranas para la interacción social más que para lo académico (Matute-Espinoza y Ochoa-Encalada, 2021), hecho que puede generar conductas de riesgo, como estar expuestos a información inadecuada con contenidos poco fiables, mala interpretación de los mensajes, difusión de información ajena al tema educativo (Rodríguez-Diaz, 2019), uso extendido en horas inoportunas, adicción a su uso (Culqui, 2020), modificaciones inadecuadas en la conducta, hábitos, valores que traen como consecuencia transformaciones en el comportamiento humano, sobre todo en los más jóvenes (Bustos y Gallardo, 2019). Fernández-De La Iglesia, (2020) recomiendan que los participantes no pasen mucho tiempo de trabajo en la aplicación, porque tiende a ser distractor frecuente y corren riesgo de dependencia o adicción; es ahí donde el docente debe cumplir un rol determinante, al estar llamado a direccionar el buen uso que los participantes hagan de estas aplicaciones online (Vizcaino-Verdú, Contreras-Pulido y GuzmánFranco, 2020).

Otro factor poco favorable para el estudiante, es la pérdida de concentración en sus labores académicas (Guerra-Torrealba, Ulloa-Erazo y Díaz-Camacho, 2018), al ser un entorno abierto y con apertura a todos sus contactos que podrían actuar como distractores; lo que hace necesario el acompañamiento familiar que apoye el trabajo docente para dar buen uso de estos espacios virtuales con fines formativos. Otro aspecto a tener en cuenta es la falta de conectividad que presentan algunos lugares donde aún persisten dificultades de acceso a la virtualidad, convirtiéndose así en un problema para las familias y los maestros; dificultades que causan retrasos con respecto al envío y recepción de mensajes, a la carga de archivos muy pesados difícil de enviar, a la interacción con los estudiantes (Calzadilla-Rodríguez, 2020).

\section{Conclusiones}

El uso de recursos tecnológicos en el ámbito educativo se ha incrementado desde el inicio de la pandemia, tanto docentes como estudiantes se 
vieron obligados a utilizar las TIC y habituarse a ellas para realizar actividades académicas. Muchas de estas tecnologías como el WhatsApp no fueron diseñadas para servir de medio a la educación, sino para otros fines muy distintos, sin embargo, de acuerdo a la revisión de la literatura realizada en este artículo, es ahora uno de los recursos tecnológicos más utilizado en la educación.

El WhatsApp evolucionó en su uso, al pasar de ser un medio de comunicación a un medio educativo que facilita la interacción entre quienes se conectan, al brindar diversas bondades y permitir la construcción de conocimiento tanto individual como grupal; así como el vincular diversas herramientas tecnológicas a través de ella. Así mismo, su incursión ha sido bastante acogida, al no requerirse un móvil sofisticado, ni requerir un ancho de banda alto, por lo que muchos docentes e instituciones educativas lo utilizan dentro del proceso enseñanzaaprendizaje, por su fácil uso, acceso y vinculación a otros recursos de web 2.0 o más. En cuanto a las funcionalidades académicas que posee el WhatsApp se destacan el poder implementar chats grupal e individual, mensajería instantánea, mensajes de voz, llamadas y videollamadas, y compartir documentos, fotos, imágenes, videos, audios, vincular páginas.

Muchas son las ventajas que se han encontrado en lo educativo, como la rapidez para la retroalimentación individual y grupal, el compartir información de interés y situada para el logro de los objetivos de la asignatura, la promoción de la participación de estudianteestudiante, docente-estudiante, la lectura de diferentes formatos de textos, la posibilidad de dejar clases grabadas o indicaciones puntuales en audio, texto, así como las evaluaciones diagnósticas, o el debate de un tema en específico; ventajas que dependen de cómo el docente organice su uso educativo.

Como desventajas latentes para los estudiantes, es el riesgo que se tiene de ser un distractor debido a su acceso abierto, así como el no contar con la conectividad en lugares donde la señal no es óptima, lo que limita el acceso a contenidos o actividades, el envío de archivos demasiados pesados o en simultaneo, lo que puede afectar la comunicación o el rol que se quiere que cumpla el WhatsApp.

Se sugiere para futuros estudios de revisión documental sobre el WhatsApp, el análisis de las herramientas que más se utilizan para el proceso de enseñanza aprendizaje de este medio tecnológico.

\section{Agradecimiento}

Un especial agradecimiento por el apoyo al desarrollo de este estudio, como parte del proyecto PIPD, Innovación docente y uso de las Tecnologías de la Información y Comunicación en el proceso de enseñanza aprendizaje con Resolución de Dirección Académica No. 012 2020-DA-UCV. Coordinación del Doctorado en Educación en la Modalidad Semipresencial de la Escuela de Posgrado de la Universidad César Vallejo.

\section{Referencias Bibliográficas}

Alfarah, M., y Bosco, A. (2018). Los Usos de Facebook y WhatsApp en la Reconstrucción de la Educación en Zonas Afectadas por Conflictos Armados: El Caso de Siria. REICE. Revista Iberoamericana sobre Calidad, Eficacia y Cambio en Educación, 16(4), 45-62.

https://doi.org/10.15366/reice2018.16.4.003

Bustos, A. R., y Gallardo, A. S. (2019). Relación entre autoestima y uso de las redes sociales Facebook y WhatsApp en estudiantes de octavo a décimo año de Educación General Básica en una Unidad Educativa Femenina Fiscomisional en Guayaquil en el periodo 2018-2019 (Tesis de pregrado). Universidad Católica de Santiago de Guayaquil, Guayaquil, Ecuador. http:// repositorio.ucsg.edu.ec/handle/3317/12865

Calzadilla-Rodríguez, I., y Ricardo-Luis, R. (2020). Tutoría de tesis de pregrado desde WhatsApp, asunción necesaria que deja COVID-19. Alcance, 9(24), 107-127. https:// orcid.org/0000-0003-1183-2868

Cascales-Martínez, A., Gomariz-Vicente, M. 
A., y Simón, A.P. (2020). WhatsApp como herramienta educativa en Educación Primaria: alumnado, docentes y familias. Pixel Bit, Revista de Medios y Educación, 58, 71-89. https://doi. org/10.12795/pixelbit.74213

Colmenares. F.S., Benavides, A., Pozo, J. y Correa, M. (2021). WhatsApp como herramienta de aprendizaje en la Enseñanza médica. In cibamanz2021. https:/cibamanz2021.sld.cu/ index.php/cibamanz/cibamanz2021/paper/ viewFile/178/117

Corona S., C. F. (2020). Facebook y otros recursos de la web 2.0 en la enseñanza aprendizaje de la electrocardiografía. Revista Cubana de Educación Médica Superior, 34(2). http://scielo.sld.cu/ scielo.php?script $=$ sci_arttext $\&$ pid $=$ S0864 $1412020000200005 \& \operatorname{lng}=$ es\&tlng=en

Crisol-Moya, E., Herrera-Nieves, L., \& MontesSoldado, R. (2020). Educación Virtual Para Todos: Una Revisión Sistemática. EUSAL Revistas, 21, 1-15. https://doi.org/10.14201/eks.20327

Culqui, R. M. (2020) Beneficios del uso del WhatsApp en estudiantes de educación secundaria en el Perú (Tesis de pregrado). Universidad Católica de Trujillo Benedicto XVI, Trujillo, Perú. http://190.223.196.26:8080/ handle/123456789/988

Escobar-Mamani, F., y Gómez-Arteta, I. (2020) WhatsApp para el desarrollo de habilidades comunicativas orales y escritas en adolescentes peruanos. Revista Comunicar, 28(65), 111-120. https://doi.org/10.3916/C65-2020-10

Escofet, A. (2020). Aprendizaje-servicio y tecnologías digitales: ¿una relación posible? RIED. Revista Iberoamericana de Educación a Distancia, 23(1), 169-182. https://doi. org/10.5944/ried.23.1.24680

Fernández-De La Iglesia, J. D. C., Casal-Otero, L., Fernández-Morante, C., y Cebreiro B. (2020). Actitudes y uso del internet $\mathrm{y}$ redes sociales en estudiantes universitarios/as de Galicia: Implicaciones personales y sociales. Revista Prisma, 28, 145-160. http://search.ebscohost. com/login.aspx? direct $=$ true $\& d b=$ fua $\& A N=1420$ 56345\&lang=es\&site $=$ eds-live.

Fitri-Rahmadi, I. (2020). WhatsApp Group for
Teaching and Learning in Indonesian Higher Education What's Up?. International Journal of Interactive Mobile Technologies 14(13). https://doi.org/10.3991/ijim.v14i13.14121 Gamero, R. V. (2020). Influencia del uso de las redes sociales en el Rendimiento Académico de los Estudiantes de $4^{\circ}$ Y $5^{\circ}$ año de Educación Secundaria de la Institución Educativa No 40164 José Carlos Mariátegui, Arequipa, 2016 (Tesis de maestría). Universidad Andina Néstor Cáceres Velásquez, Arequipa, Perú. http://repositorio. uancv.edu.pe/handle/UANCV/4698

Guerra-Torrealba, L., Ulloa-Erazo, N., y DíazCamacho, E. (2018). Uso ético de las redes sociales - hacia la Educación para la competitividad. CISTI (Iberian Conference on Information Systems y Technologies, 1-5.

Lafaurie-Molina A. M., Sinning-Ordóñez P. A., y Valencia-Cobo J. A. (2018). WhatsApp y Facebook como mediación pedagógica en procesos de Orientación Socio Ocupacional. Educación y Educadores, 21(2), 179-199. https:// doi.org/10.5294/edu.2018.21.2.1

Maldonado-Berea, G. A., García-Gonzales, J., y Sampedro-Requena, B. E. (2019). El efecto de las TIC y redes sociales en estudiantes universitarios. RIED: Revista Iberoamericana de Educación a Distancia, 22(2), 153.

https://doi.org/10.5944/ried.22.2.23178

Malo-Cerrato, S., Martin-Perpina, M.M., y Vinas Poch, F. (2018). Uso excesivo de redes sociales: Perfil psicosocial de adolescentes españoles. Comunicar, 56, 101. https://doi.org/10.3916/ C56-2018-10

Marín-Díaz, V., y Cabero-Almenara, J. (2019). Las redes sociales en educación: desde la innovación a la investigación educativa. RIED. Revista Iberoamericana de Educación a Distancia, 22(2), 25-33. https://doi.org/10.5944/ ried.22.2.24248

Mas, J. (2019). Museos españoles en Facebook: análisis de su comunicación en el marco del museo social digital José Manuel (Tesis de postgrado). Universidad Carlos III de Madrid, España. https://e-archivo.uc3m.es/ handle/10016/28564\#preview Matute-Espinoza, M. P., y Ochoa-Encalada, S. C. 
(2021). WhatsApp y Messenger como estrategia para el aprendizaje de niños de Educación Básica. CIENCIAMATRIA, 7(13), 322-344.

Mayor-Buzón, V., García-Pérez, R., y RebolloCatalán, M.A. (2019). Explorando factores predictores de la competencia digital en las redes sociales virtuales. Pixel-Bit: Revista de Medios y Educación, 56, 51-69.

https://doi.org/10.12795/pixelbit.2019.i56.03

Mejía, J. G. (2019). Efectividad de la implementación de las tecnologías de la información y la comunicación (TIC), en el ámbito educativo (Tesis de maestría). Universidad de la Sabana, Chía, Colombia. http://hdl.handle. net/10818/36317

Molina-García, M. J. (2020). ¿Hablamos? Análisis del discurso y estrategias empleadas en Whatsapp por estudiantes universitarios. Onomázein, 274294.

https://doi.org/10.7764/onomazein.ne6.14

Montilla, Y. N. (2020). WhatsApp como herramienta educativa en la enseñanza aprendizaje. Revista Vinculando. https:// vinculando.org/beta/whatsapp-comoherramienta-educativa-en-la-ensenanzaaprendizaje.html\#vcite

Moreno, B., Muñoz M., Cuellar J., Domancic S., y Villanueva J. (2018). Revisiones Sistemáticas: definición y nociones básicas. Revista clínica de periodoncia, implantología y rehabilitación oral, 11(3), 184-186. https://doi.org/10.4067/S071901072018000300184

Oliveira-Araujo, J. B., Gomes, Matheus, \& Barcellos, Thais. (2020). A Covid-19 e a volta às aulas: ouvindo as evidências. Ensaio: Avaliação e Políticas Públicas em Educação, 28(108), 555-578. https://doi.org/10.1590/s010440362020002802885

Paredes, I. L. (2020). Uso de las redes sociales: Facebook, WhatsApp, Instagram y su influencia en la ortografía de los estudiantes del quinto grado de secundaria del Colegio Unión, 2020 (Tesis de pregrado). Universidad Peruana Unión, Lima, Perú. https://repositorio.upeu.edu.pe/ handle/20.500.12840/4190

Pérez-Cruz, D., Sánchez-López, F., CocónJuárez, J. F., y Zavaleta-Carrillo, P. (2020). La
Influencia del WhatsApp en la Educación Superior de la UNACAR. Revista TecnológicaEducativa Docentes 2.0, 9(2), 39-48. https://doi. org/10.37843/rted.v9i2.143

Rodríguez-Diaz, E. (2019). Peculiaridades de las redes sociales. HETS online Journal, 10, 182-209. http://search.ebscohost.com/login.aspx?direct=t rue $\& \mathrm{db}=$ eue $\& \mathrm{AN}=140057540 \&$ lang $=\mathrm{es} \&$ site $=\mathrm{e}$ ds-live.

Román, F., Marín, D., y Peirats, J., (2021). Avances en la investigación sobre la implementación del aula invertida en primaria. Revista científica electrónica de Educación y Comunicación en la Sociedad del Conocimiento, 21(1), 141-170. https://doi.org/10.30827/eticanet.v21i1.16991 Ruvalcaba- Arredondo L., Torres-Cosío V. Alejandro-Carmona E., y Pérez-Veyna O. (2019). Perfil estudiantil: uso de WhatsApp y Facebook. NOVUM, 1(9), 32 - 57. https://revistas.unal.edu. co/index.php/novum/article/view/73101

Safiatur, R. (2020) Students' writing skill through Telecollaboration: in the context of WhatsApp and Facebook. Loquen: English Studies Journal. 30, 31-39.

https://doi.org/10.32678/loquen.v13i1.2381 Shahid, S. y Shaikh, M. A. (2019). Impact of "WhatsApp Chaupal" on the Academic Performance of Graduate Students of Karachi-A Case Study. FWU Journal of Social Sciences, 13(2), 94-107. direct $=$ true $\& d b=a 9 h \& A N=13915$ 4500\&lang $=$ es\&site $=$ eds-live

Sola-Reche, J. M., García-Vidal, M., y OrtegaNavas, M. C. (2019). Las implicaciones del uso de dispositivos móviles en el proceso de enseñanza aprendizaje en alumnos de $5^{\circ}$ Y $6^{\circ}$ de Primaria. Píxel-BIT Revista de Medios y Educación, 55, 117-131.

https://doi.org/10.12795/pixelbit.2019.i55.07

Suárez-Lantarón, B. (2018). WhatsApp: su uso educativo, ventajas y desventajas. Revista de Investigación en Educación, 16(2), 121-135. http://reined.webs4.uvigo.es/index.php/reined/ article/view/342

Tapia-Repetto, G., Gutierrez, C., y TremilloMaldonado, O. (2019). Nuevas tecnologías en educación superior. Estudio de percepción en estudiantes acerca del uso de WhatsApp y 
Entornos Virtuales de Aprendizaje (Plataforma Moodle). Odontoestomatología, 21(33), 37-43. https://doi.org/10.22592/ode2019n33a5

UNESCO (2021). A un año del comienzo de la pandemia: continuidad educativa y evaluación en América Latina y el Caribe en 2021. Organización de las Naciones Unidas para la Educación, la Ciencia y la Cultura. https://unesdoc.unesco.org/ ark:/48223/pf0000377802

Veytia-Bucheli, M. G., y Bastidas-Terán, F.A. (2020). WhatsApp como recurso para el trabajo grupal en estudiantes universitarios. Apertura: Revista de Innovación Educativa, 12(2), 74-93. https://doi.org/10.32870/Ap.v12n2.1911

Vizcaino-Verdú, A., Contreras-Pulido, P. y Guzmán-Franco, M. D. (2020). Construcción del concepto fanfullying: revisión crítica del acoso en redes sociales. Pixel-Bit: Revista de Medios y Educación. 57, 211-230.

https://doi.org/10.12795/pixelbit.2020.i57.09

WhatsApp (2021). WhatsApp. https://www. whatsapp.com/ 